\title{
Effects of Dynamic Warm-up With and Without a Weighted Vest on Lower Extremity Power Performance of High School Male Athletes
}

\author{
John Carter, Amber Boehner, Cori Cameron, Jessica Murphy, Ashley Peintner \\ Physical Therapy Department
}

\begin{abstract}
The purpose of this study was to compare lower extremity power performance utilizing the Margaria-Kalamen Power Test after a dynamic warm-up with and without a weighted vest. Sixteen $(n=16)$ high school male football players participated in two randomly ordered testing sessions. One session involved performing the football team's typical warm-up while wearing a vest weighted at $5 \%$ of the individual athlete's body weight before performing 3 trials of the Margaria-Kalamen Power Test. The second session involved performing the same team warm-up without wearing a weighted vest before performing 3 trials of the Margaria-Kalamen Power Test. The dynamic warm-up consisted of the following dynamic exercises, which lasted 5 minutes total: straight leg kicks, forward lunges, backward lunges, heel-rear kicks, high knees, stride-outs, deep lunges, and jogging. No significant difference was found in power performance between the non-resisted and resisted dynamic warm-up protocols $(p=0.161)$. It was concluded that a dynamic warm-up with a vest weighted at $5 \%$ of the athlete's body weight was not advantageous for increasing lower extremity power output in high school football players.
\end{abstract}

Keywords: power, male, weighted vest, Margaria-Kalamen Power Test

\section{Introduction}

Research has shown that by using resistance during dynamic warm-up, power performance improved in high school female athletes (1) and male/female collegiate athletes $(2,3)$. The effectiveness of a resisted dynamic warmup for high school male athletes has not yet been determined. Consequently, this study was designed to compare lower extremity power performance of high school male athletes following a dynamic warm-up without a weighted vest and dynamic warm-up with a vest weighted at $5 \%$ of the athlete's body weight. Using previous research to guide our exploration of power production in athletic performance, we hypothesized that the use of a weighted vest during dynamic warm-up protocols would amplify lower extremity power performance in high school male athletes to a greater degree than dynamic warm-up activities without a weighted vest.

The information ascertained by this study is significant for the progression of practice, training, and competition warm-up protocols in the augmentation of power performance of high school male athletes and could be of benefit to coaches, athletic trainers, athletes, and health care professionals.

\section{Experiment, Results, Discussion, and Significance}

Participants who sustained a lower extremity injury that required surgical intervention in the two years prior to testing were excluded from the study. Subjects were also required to have obtained a current physical allowing them to participate in athletic events, which provided us with additional evidence that each participant was healthy enough to participate in the study. Sixteen male athletes, 14 to 18 years old, who were members of a local high school's football team and met the original criteria volunteered for this study. Participants served as their own controls.

Each subject was required to participate on two separate testing days which were exactly one week apart. On the initial testing day, the subjects were randomly divided into two groups. On Day 1, Group A wore weighted vests during the dynamic warm-up and Group B did not wear vests during the warm-up. Both groups completed a standardized dynamic warm-up together. Following the warm-up, Group A removed the weighted vests. Groups A and $\mathrm{B}$ combined into one large group for the power testing procedures and moved to a staircase in a gymnasium to perform the Margaria-Kalamen Power Test. On Day 2, Group B wore weighted vests during the dynamic warm-up and Group A did not wear vests during the warm-up. Since all sessions involved the same warm-up and the power 
testing was performed in the same gymnasium on the same staircase, inter-day reliability of the testing procedures was established.

A paired $t$-test was used to determine whether there was a significant difference between mean power values after warm-ups with and without a weighted vest. A two-tailed test was conducted with alpha set at .05 . The paired $t$-test was used because there was one independent variable (type of warm-up) with two related or dependent samples (with and without a weighted vest) and one dependent variable (power) in this study. The test was twotailed because the hypothesis was non-directional.

The paired $t$-test showed that there was no significant difference $\left(\mathrm{t}_{15}=-1.474, \mathrm{p}=0.161\right)$ between warm-up protocols. The mean difference in absolute power (with - without weighted vest) was $-80.50+/-218.5 \mathrm{~W}$. There was, however, considerable intersubject variability (755.1 W to $1814.4 \mathrm{~W})$.

Major limitations to our study may have included the lack of available resources (time, space, participants), group testing rather than individual testing, inappropriate sensitivity of the timing mats and specificity of the Margaria-Kalamen Power Test, inability to control the effort put forth by the participants, and either insufficient resistance provided by the weighted vests or excessive resistance leading to fatigue of the lower extremities (1).

Our findings are consistent with some $(1,4)$, but not all (1-3) research comparing the effects of different warmup protocols on power performance. Future research related to this topic should redevelop and expand upon the foundations of our study and could include diversified dynamic warm-up protocols, different tests of lower extremity power performance (i.e. standard vertical jump, long jump, 10 yard sprint, Wingate Test), multiple warmup durations, and varying resistances. In addition, prospective studies could include a more assorted sample, comprised of male and female participants of varying age, training status, and athletic background. Individual testing sessions may be beneficial, as well, to reduce the effects of time-dependent power change, camaraderie, intersubject distraction, and a learning curve.

\section{Conclusions}

Our study found no significant difference when comparing lower extremity power performance of high school male football players following a dynamic warm-up without a weighted vest and dynamic warm-up with a vest weighted at $5 \%$ of the athlete's body weight, utilizing the Margaria-Kalamen Power Test. These findings are consistent with some $(1,4)$, but not all (1-3) research comparing the effects of different warm-up protocols on power performance. According to our findings, a resisted dynamic warm-up does not enhance power performance to a greater degree than a non-resisted dynamic warm-up in high school male athletes.

\section{Acknowledgements}

We would like to acknowledge: Valley Center High School, USD 262, Dr. Louise Herrington VCHS Principal, Mr. Jamie Lewis and Mr. Brian Gregoire VCHS Assistant Principals, Mr. Vic Noordhoek VCHS Athletic Director, Coach Jon Black and the Valley Center High School 2008 Football Team. We would also like to thank Dr. Barb Smith, our statistician, and the Delano Maggard, Jr. Graduate Research Grant for funding.

[1] Faigenbaum AD, McFarland JE, Schwerdtman JA, Ratamess NA, Kang J, Hoffman JR. Dynamic warm-up protocols, with and without a weighted vest, and fitness performance in high school female athletes. $J$ Athl Train. 41:357-363. 2006.

[2] Burkett LN, Phillips WT, Ziuraitis J. The best warm-up for the vertical jump in college-age athletic men. J Strength Cond Res. 19:673-676. 2005.

[3] Thompsen, AG, Kackley T, Palumbo MA, Faigenbaum AD. Acute effects of different warm-up protocols with and without a weighted vest on jumping performance in athletic women. J Strength Cond Res. 21:52-56. 2007.

[4] Koch AJ, O'Bryant HS, Stone ME, Sanborn K, Proulx C, Hruby J, Shannonhouse E, Boros R, Stone MH. Effect of warm-up on the standing broad jump in trained and untrained men and women. J Strength Cond Res. 17:710-714. 2003. 